\title{
Yttrium-90 radioembolization of unresectable hepatocellular carcinoma - a single center experience
}

\author{
Carsten Meyer ${ }^{1, *}$ \\ Claus Christian Pieper ${ }^{1, *}$ \\ Hojjat Ahmadzadehfar ${ }^{2}$ \\ Nina Alexandra Lampe' \\ Eva Maria E Matuschek' \\ Thomas Adrian Maschke' \\ Simon Jonas Enkirch' \\ Markus Essler ${ }^{2}$ \\ Ulrich Spengler ${ }^{3}$ \\ Hans Heinz Schild' \\ 'Department of Radiology, University \\ of Bonn, Sigmund-Freud-Strasse, \\ Bonn, Germany, ${ }^{2}$ Department of \\ Nuclear Medicine, University of \\ Bonn, Sigmund-Freud-Strasse, \\ Bonn, Germany, ${ }^{3}$ Department of \\ Internal Medicine I, University \\ of Bonn, Sigmund-Freud-Strasse, \\ Bonn, Germany \\ *These authors contributed equally \\ to this work
}

This article was published in the following Dove Press journal:

OncoTargets and Therapy

26 September 2017

Number of times this article has been viewed

Purpose: To determine the value of radioembolization (RE) for treatment of unresectable hepatocellular carcinoma (HCC).

Patients and methods: Records of patients undergoing RE for unresectable HCC were retrospectively reviewed. Biochemical and clinical toxicities, imaging response (according to modified Response Evaluation Criteria In Solid Tumors), time-to-progression (TTP) and overall survival (OS) were analyzed. Data were stratified according to clinical and procedural parameters. Univariate and multivariate analyses were performed.

Results: One hundred and fifteen patients ( 89 male, mean age 69.3 years) underwent 158 REs (119 resin-, 39 glass-based) (Barcelona Clinic Liver Cancer [BCLC]-A: 6.1\%, B: 33.9\%, C: $60.0 \%)$. Median clinical follow-up was $5.9(0.9-83.5)$ months. No grade 4 or 5 clinical toxicities were noted. Objective response rate was $35.6 \%$; disease control rate was $76.7 \%$. Median TTP of the treated part of the liver was $4(0.9-45.4)$ months. 108/115 patients died during follow-up (median OS 8.4 [0.3-82.8] months after first RE [BCLC-A: 52.8 months, BCLC-B: 12.4 months, BCLC-C: 6.1 months]). On multivariate analysis, baseline Eastern Co-operative Oncology Group status $<1$, ascites prior to RE and best imaging response were predictors of longer OS. In BCLC-C patients, tumor burden, ascites prior to RE, baseline gamma-glutamyltransferase and Child-Pugh score were predictive of OS.

Conclusions: RE is safe and effective in carefully selected patients suffering from HCC with a low complication rate. Low baseline Eastern Co-operative Oncology Group status and absence of ascites prior to RE are positive prognostic factors.

Keywords: radioembolization, hepatocellular carcinoma, HCC, locoregional therapy, liver

\section{Introduction}

Hepatocellular carcinoma (HCC) is the most common primary hepatic malignancy and one of the leading causes of cancer-related death worldwide. ${ }^{1,2}$

Complete resection, liver transplantation or - within certain limits - ablative therapies as the only curative treatment options are feasible in just $30 \%$ of patients. ${ }^{2}$ In early and intermediate stage $\mathrm{HCC}$, loco-regional treatments like radiofrequency ablation or transarterial chemoembolization (TACE) or combination thereof are frequently employed. ${ }^{3-11}$ In advanced HCC, systemic molecular targeted therapy with sorafenib has demonstrated a statistically significant, but limited increase in overall survival (OS), though this is achieved with a rather high rate of adverse events. ${ }^{12-14}$ Alternative treatment options are therefore necessary, especially in cases of advanced HCC associated with portal vein thrombosis, which is associated with a particularly poor prognosis (median survival $2-4$ months with best supportive care). ${ }^{15-17}$
Correspondence: Claus Christian Piepe Department of Radiology, University of Bonn, Sigmund-Freud-Str 25, 53105 Bonn, Germany Tel +49 228 287| 5870 Fax +49228287 I 5598 Email claus.christian.pieper@ukb. uni-bonn.de 
Radioembolization (RE) has been introduced as an alternative treatment for intermediate and advanced stage $\mathrm{HCC}^{18}$ and was already shown to result in longer timeto-progression (TTP) compared with conventional TACE in early and intermediate stage HCC. ${ }^{19}$ The aim of the present study was to determine the value of RE in the treatment of unresectable HCC across a range of tumor stages and to determine prognostic parameters.

\section{Patients and methods}

\section{Patients and data acquisition}

Patients suffering from HCC undergoing RE at our institution between 09/2006 and 02/2015 were identified in the clinical database. Indications to perform RE were discussed in interdisciplinary consensus conferences in accordance with published indications for RE..$^{20,21}$ Typical indications for $\mathrm{RE}$ at our institution were progressive disease (PD) under a TACE treatment regime, contraindications for TACE/ chemotherapy (eg, portal vein thrombosis/invasion, cardiac comorbidities) as well as impaired hemostasis (as RE usually only requires 2 angiography sessions compared with often multiple TACE sessions). All patients presented with HCC that was deemed unresectable by an experienced liver surgeon. Repeated RE of the same part of the liver was offered to selected patients showing disease progression after primary RE in interdisciplinary consensus according to the individual clinical situation of the patient. The study was approved by the local institutional review board of the University Hospital of Bonn; informed patient consent was waived due to the retrospective character of the study.

Study inclusion criteria were histologically confirmed HCC or hepatic malignancy showing imaging characteristics typical of HCC (according to the EASL-EORTC criteria $^{22}$ ), RE performed at our institution and accessible patient data. Baseline clinical and laboratory parameters, procedural data and results of follow-up examinations were retrospectively reviewed. Baseline cross-sectional imaging was used to estimate the relative liver tumor burden as $<25 \%$, $25 \%-50 \%$ or $>50 \%$.

\section{Treatment}

Pre-treatment workup, including clinical/laboratory examination and cross-sectional imaging (preferentially magnetic resonance imaging [MRI] of the liver), was performed according to clinical standards. ${ }^{21,23}$ Pre-treatment catheter angiography with injection of Technetium-99m macroaggregated albumin into the target arteries followed by single photon emission computed tomography/CT was performed to exclude non-target sphere deposition. Vessels supplying extrahepatic tissue originating from the hepatic artery were coil-embolized at the discretion of the interventionalist. Treatment activity was calculated in compliance with international consensus guidelines ${ }^{21}$ (body surface area [BSA] method for resin microspheres [SIR spheres, Sirtex Medical Limited, North Sydney, Australia] and the MIRD equation as provided by the manufacturer for glass microspheres [TheraSphere, BTG, London, UK]). RE was performed either in a single session (simultaneous bilobar or unilobar) or a sequential lobar approach depending on tumor distribution and liver function using a microcatheter (eg, Renegade, Boston Scientific, Natick, MA, USA). Glass microspheres were generally preferred in cases with portal vein thrombosis (especially if the main portal vein was affected), in patients with unilobar disease who were planned to received unilobar RE without the need for catheter repositioning, as well as for repeat RE. Peri-interventional medication included dexamethasone, ondansetron and pantoprazole. All patients were admitted to a special ward for 2 days after RE, in accordance with local radioprotection regulations of the Federal Office for Radiation Protection in Germany (BfS).

\section{Toxicities}

The Common Terminology Criteria for Adverse Events of the National Cancer Institute (CTCAE v. 4.03) were used for assessment of toxicities of biochemical parameters both before (baseline) and after RE (bilirubin, aspartate aminotransferase [AST], alanine aminotransferase, gamma glutamyl transferase [GGT]), the presence and development of ascites and clinical adverse events. Procedure-related clinical complications, for example, gastroduodenal ulceration due to non-target embolization, were noted.

\section{Follow-up regime}

Patients underwent 2 early follow-up examinations 4-6 weeks and 3 months after RE, including physical examination, laboratory liver function tests and contrast-enhanced standard MRI or CT of the liver. After that, follow-up examinations at 3-month intervals were recommended.

\section{Hepatic response}

Imaging response was assessed by a radiologist, blinded to patients' overall outcome, on arterial-phase cross-sectional imaging according to modified Response Evaluation Criteria In Solid Tumors (mRECIST) criteria. ${ }^{24}$ Two target lesions were selected in cases of single session whole liver treatment. In patients receiving sequential lobar or unilobar treatment, 
2 lesions per lobe were selected to separately assess treatment response for the treated and non-treated liver lobe. Best imaging response (best response to treatment over the entire follow-up period) is reported separately for single session treatments (simultaneous bilobar or unilobar) and for sequential bilobar treatment (after completion of the treatment cycle) as well as for repeated RE of previously treated tissue.

\section{Definitions and statistical analysis}

OS was defined as the time between the first RE procedure performed in a patient and death of any cause. TTP was defined as the time between RE and first detected progression and was calculated separately for treated (time to hepatic progression $[\mathrm{TTHP}]_{\text {treated }}$ ) and untreated parts of the liver $\left(\right.$ TTHP $\left._{\text {untreated }}\right)$ as well as for extrahepatic progression (time to extrahepatic progression [TTEP]) (first detection of new extrahepatic lesions or increase of the long axis of an existing lesion by $>20 \%$ ).

Statistical analyses were performed using commercially available statistical software (SPSS, version 22.0, IBM, Armonk, NY, USA). Univariate Kaplan-Meier analysis with a log-rank test for statistical significance was performed to evaluate associations of OS with clinical parameters for categorical parameters. A Cox regression model was used for continuous variables. Stepwise multivariate Cox regression was then performed to identify independent predictors of OS and to calculate hazard ratio (HR) estimates for all parameters showing significant associations on univariate analysis. All analyses were performed for the whole group and the subgroups of patients with Barcelona Clinic Liver Cancer (BCLC) stage B and C separately.

\section{Results \\ Patient characteristics}

Overall 115 patients ( 89 male, mean age $69.3 \pm 10.6$ years) underwent RE for unresectable HCC. Detailed patient characteristics are given in Table 1. A total of $158 \mathrm{RE}$ procedures were performed with 18 patients receiving single session whole liver treatment (16 resin-, 2 glass-based REs; 10 with lobar sphere application, 8 with application via the proper hepatic artery), 78 patients receiving unilobar treatment (56 resin, 22 glass) and 19 patients with sequential bilobar treatment (either resin [ $\mathrm{n}=30$ ] or glass $[\mathrm{n}=8]$ ) (median time between procedures 2.5 [0.9-32.2] months). Mean administered activities are given in Table 1. In sequential bilobar treatment, cumulative activity was $3.19 \pm 1.24 \mathrm{GBq}$. Twenty-one patients received a second RE of the treated part of the liver (16 resin, 5 glass); 3 patients received a third RE (1 resin, 2 glass) (median time between primary and repeated procedures 5.5 [1.2-44.8] months).

Median time between baseline imaging and RE was $0.7(0-5.3)$ months. Twenty-five patients were lost to imaging follow-up, 5 of these did not survive until first follow-up or were terminally ill at that time. Median imaging follow-up was 5.9 (0.9-61.6) months. Clinical follow-up was available in 103/115 patients (median clinical follow-up 5.9 [0.9-83.5] months). Six out of 115 patients were alive on the date of analysis (median time after RE 17.6 [14.9-82.8] months). The status of 1 patient was unknown.

\section{Toxicities}

No access-related complications were observed. During RE, dissection of the right hepatic artery occurred in 2 cases without the need for treatment. Three patients $(2.6 \%)$ presented with grade 2 gastroduodenal ulceration ( 1 after whole liver, 2 after right lobar treatment). The gastroduodenal artery, but not the right gastric artery was previously embolized in all 3 cases. One of the 3 cases of gastroduodenal ulceration occurred in a patient showing severe blood-flow stasis (only $55 \%$ of the intended activity administered). In 1 patient, dyspnea developed 2 months after RE, so radiation-induced pneumonitis was suspected. The patient, however, did not require any treatment. One patient developed septicemia 2 days after completion of a sequential bilobar RE cycle and died 7 days later. This patient did not present with dilated bile ducts or signs of cholangitis. Otherwise, no grade 4 or 5 clinical toxicities were noted.

During follow-up, 50 patients developed new or worsening ascites (all $\leq$ grade 2); 25 of these cases were observed within the first 3 months after RE (after sequential RE: 6/18 [33.3\%]; single session bilobar RE: 5/19 [26.3\%], unilobar RE: 8/78 [10.3\%], repeated RE: 6/21 [28.6\%]).

Biochemical toxicities are summarized in Tables 2 and 3. After RE, the majority of patients showed no or only grade $\leq 2$ toxicities in investigated laboratory parameters. Patients receiving sequential lobar treatment showed a permanent increase in bilirubin levels in $61.1 \%(11 / 18)$, while patients receiving whole liver treatment in a single session or unilobar treatment showed permanent changes in only about $29 \%$ (21/72) of cases. Otherwise, changes in laboratory toxicity levels were comparable between the different treatment strategies. Of note, GGT levels were already elevated to grade 3 or 4 in 52/109 patients at baseline. After repeated RE, $8 / 21$ patients (38.1\%) showed an increase from grade 1 or 2 to grade 3 or 4 bilirubin toxicity. 
Table I Patient baseline characteristics and procedural data

\begin{tabular}{|c|c|c|c|c|}
\hline & Overall & BCLC-A & BCLC-B & BCLC-C \\
\hline Patients & 115 & 7 & 39 & 69 \\
\hline \multicolumn{5}{|l|}{ Gender (\%) } \\
\hline Female & $29(25.2)$ & I (I4.3) & II (28.2) & $17(24.6)$ \\
\hline Male & $86(74.8)$ & $6(85.7)$ & $28(7 \mid .8)$ & $52(75.4)$ \\
\hline \multirow[t]{2}{*}{ Mean age, (years; range) } & $69.3 \pm 10.6$ & $72.7 \pm 12.1$ & $69.4 \pm 10.7$ & $68.8 \pm 10.6$ \\
\hline & $(34.8-90.0)$ & $(54.2-86.4)$ & $(40.5-82.3)$ & $(34.8-90.0)$ \\
\hline Time between first diagnosis of & $14.9 \pm 26.0$ & $26.4 \pm 34.2$ & $17.3 \pm 35.4$ & $12.0 \pm 16.3$ \\
\hline HCC and RE (months) ( $\mathrm{n}=99)$ & $(0.3-198.4)$ & $(1.9-101.5)$ & $(0.8-198.4)$ & $(0.3-84.1)$ \\
\hline \multicolumn{5}{|l|}{ ECOG status $(n=84)(\%)$} \\
\hline 0 & $38(45.2)$ & $4(80)$ & $19(61.3)$ & $15(31.3)$ \\
\hline I & $30(35.7)$ & $0(0)$ & $7(22.6)$ & $23(47.9)$ \\
\hline 2 & $16(19.1)$ & I (20) & $5(16.1)$ & $10(20.8)$ \\
\hline \multicolumn{5}{|c|}{ Estimated relative liver tumor burden $(n=|| 3)$} \\
\hline$<25 \%$ & $53(46.9)$ & $6(100)$ & $23(59.0)$ & $24(35.3)$ \\
\hline $25 \%-50 \%$ & $50(44.2)$ & $0(0)$ & $14(35.9)$ & $36(52.9)$ \\
\hline$>50 \%$ & $10(8.9)$ & $0(0)$ & $2(5.1)$ & $8(11.8)$ \\
\hline \multicolumn{5}{|l|}{ Pattern of tumor manifestation (\%) } \\
\hline Solitary & $18(15.7)$ & $4(57.1)$ & $6(15.4)$ & $8(11.6)$ \\
\hline Oligofocal & $19(16.5)$ & $3(42.9)$ & $7(17.9)$ & $9(13.0)$ \\
\hline Multifocal & $72(62.6)$ & $0(0)$ & $26(66.7)$ & $46(66.7)$ \\
\hline Diffuse & $6(5.2)$ & $0(0)$ & $0(0)$ & $6(8.7)$ \\
\hline \multicolumn{5}{|l|}{ Distribution of metastases (\%) } \\
\hline Unilobar right & $28(24.4)$ & $5(71.4)$ & $7(18.0)$ & $14(20.3)$ \\
\hline Unilobar left & $9(7.8)$ & $0(0)$ & $5(12.8)$ & $4(5.8)$ \\
\hline Bilobar & $78(67.8)$ & $2(28.6)$ & $27(69.2)$ & $5 I(73.9)$ \\
\hline \multicolumn{5}{|l|}{ Liver cirrhosis (\%) } \\
\hline Yes & $86(74.8)$ & $6(85.7)$ & $31(79.5)$ & $49(71.0)$ \\
\hline No & $29(25.2)$ & I (14.3) & $8(20.5)$ & $20(29.0)$ \\
\hline \multicolumn{5}{|l|}{ Cause of cirrhosis $(n=86)(\%)$} \\
\hline Hepatitis & $37(43.0)$ & $2(40)$ & $10(32.3)$ & $25(50)$ \\
\hline Alcohol & $49(57.0)$ & $3(60)$ & $21(67.7)$ & $25(50)$ \\
\hline \multicolumn{5}{|l|}{ Child-Pugh-status (\%) } \\
\hline$A$ & $104(90.4)$ & $7(100)$ & $39(100)$ & $58(84.1)$ \\
\hline B & II (9.6) & $0(0)$ & $0(0)$ & II (I5.9) \\
\hline \multicolumn{5}{|l|}{$\mathrm{BCLC}(\%)$} \\
\hline$A$ & $7(6.1)$ & & & \\
\hline B & $39(33.9)$ & & & \\
\hline C & $69(60.0)$ & & & \\
\hline Mean MELD score & $8.4 \pm 2.4(5-20)$ & $6.9 \pm 2.0(5-10)$ & $7.9 \pm 2.1(5-13)$ & $9.1 \pm 2.5(6-20)$ \\
\hline \multicolumn{5}{|l|}{ Ascites prior to RE ( $n=|| 2)(\%)$} \\
\hline No & $86(76.8)$ & $6(100)$ & $34(89.5)$ & $46(67.7)$ \\
\hline Minimal & $23(20.5)$ & $0(0)$ & $4(10.5)$ & $19(27.9)$ \\
\hline Extensive & $3(2.7)$ & $0(0)$ & $0(0)$ & $3(4.4)$ \\
\hline \multicolumn{5}{|l|}{ Extrahepatic disease (\%) } \\
\hline Overall & $43(37.4)$ & $0(0)$ & $0(0)$ & $43(62.3)$ \\
\hline Nodal & $28(24.3)$ & $0(0)$ & $0(0)$ & $28(40.6)$ \\
\hline Pulmonary & $13(11.3)$ & $0(0)$ & $0(0)$ & $13(18.8)$ \\
\hline Osseous & $7(6.1)$ & $0(0)$ & $0(0)$ & $7(10.1)$ \\
\hline Brain & $0(0)$ & $0(0)$ & $0(0)$ & $0(0)$ \\
\hline Others & $\begin{array}{l}8 \text { ( } 2 \text { cutaneous, } 2 \text { peritoneal, } \\
2 \text { adrenal, I pancreatic, } \\
\text { I diaphragm infiltration) }\end{array}$ & $0(0)$ & $0(0)$ & $8(11.6)$ \\
\hline \multicolumn{5}{|l|}{ Vascular invasion (\%) } \\
\hline Overall & $29(25.2)$ & $0(0)$ & $0(0)$ & $29(42.0)$ \\
\hline Portal vein & $26(22.6)$ & $0(0)$ & $0(0)$ & $26(37.7)$ \\
\hline Hepatic vein & $2(1.7)$ & $0(0)$ & $0(0)$ & $2(2.9)$ \\
\hline Both & I (0.9) & $0(0)$ & $0(0)$ & I (I.4) \\
\hline
\end{tabular}


Table I (Continued)

\begin{tabular}{|c|c|c|c|c|}
\hline & Overall & BCLC-A & BCLC-B & BCLC-C \\
\hline \multicolumn{5}{|l|}{ Previous liver resection (\%) } \\
\hline Overall & $21(18.3)$ & $3(42.9)$ & $8(20.5)$ & $10(14.5)$ \\
\hline Atypical & $7(6.1)$ & I (I4.3) & $2(5.1)$ & $4(5.8)$ \\
\hline Segmental & $8(7.0)$ & $0(0)$ & $4(10.3)$ & $4(5.8)$ \\
\hline Left hemihepatectomy & $2(1.7)$ & $0(0)$ & I (5.I) & I ( 1.4$)$ \\
\hline Right hemihepatectomy & $4(3.5)$ & $2(28.6)$ & I $(5.1)$ & I ( 1.4$)$ \\
\hline \multicolumn{5}{|c|}{ Previous application of sorafenib $(n=|| 3)(\%)$} \\
\hline Yes & $24(21.2)$ & I (I4.3) & 7 (I7.9) & $16(23.9)$ \\
\hline No & $89(78.8)$ & $6(85.7)$ & $32(82.1)$ & $5 I(76.1)$ \\
\hline \multicolumn{5}{|l|}{ Previous TACE (\%) } \\
\hline Overall & $31(27.0)$ & $2(28.6)$ & $15(38.5)$ & $14(20.3)$ \\
\hline IX & $14(12.2)$ & $I(14.3)$ & $8(20.5)$ & $5(7.3)$ \\
\hline $2 \times$ & $3(2.6)$ & $0(0)$ & $2(5.1)$ & I (I.4) \\
\hline$>2 x$ & $14(12.2)$ & I (I4.3) & $5(12.8)$ & $8(11.6)$ \\
\hline No & $84(73.0)$ & $5(71.4)$ & $24(61.5)$ & $55(79.7)$ \\
\hline Mean baseline bilirubin $(\mathrm{mg} / \mathrm{dL})$ & $1.0 \pm 0.6$ & $0.8 \pm 0.5$ & $0.8 \pm 0.4$ & $1.1 \pm 0.7$ \\
\hline$(n=I I I)$ & $(0.10-3.6)$ & $(0.3-1.6)$ & $(0.1-1.4)$ & $(0.3-3.6)$ \\
\hline Mean baseline ALT (U/L) & $57.4 \pm 47.2$ & $58.3 \pm 42.4$ & $42.1 \pm 26.7$ & $65.3 \pm 54.1$ \\
\hline$(n=\mid 12)$ & $(12-325)$ & $(20-140)$ & $(13-126)$ & $(12-325)$ \\
\hline Mean baseline AST (U/L) & $88.6 \pm 79.5$ & $112.4 \pm 150.0$ & $57.9 \pm 41.8$ & $102.5 \pm 81.7$ \\
\hline$(n=I I I)$ & $(15-440)$ & $(23-440)$ & $(15-257)$ & $(18-372)$ \\
\hline Mean baseline GGT (U/L) & $349.0 \pm 608.7$ & $132.7 \pm 87.5$ & $30 I .4 \pm 276.6$ & $396.4 \pm 747.9$ \\
\hline$(n=I I I)$ & $(35-6, \mid 88)$ & $(46-274)$ & $(37-I, \mid 89)$ & $(35-6,188)$ \\
\hline Mean baseline albumin & $34.8 I \pm 5.4 I$ & $37.6 \pm 6.0$ & $37.5 \pm 4.9$ & $33.3 \pm 5.1$ \\
\hline$(\mathrm{mg} / \mathrm{dL})(\mathrm{n}=90)$ & $(20.70-47.00)$ & $(31.1-47.1)$ & $(20.7-44.5)$ & $(21.9-45.2)$ \\
\hline Mean intended $\mathrm{Y} 90$ activity & $1.69 \pm 0.74 /$ & $1.43 \pm 0.78 /$ & $1.57 \pm 0.80 /$ & $1.80 \pm 0.67 /$ \\
\hline$($ resin $[n=I 17] /$ glass [n=39]) (GBq) & $2.03 \pm 0.95$ & $2.00 \pm 0.0$ & $2.22 \pm 1.29$ & $1.95 \pm 0.83$ \\
\hline Mean administered $Y 90$ activity & I.64 $60.76 /$ & $1.43 \pm 0.48 /$ & $\mathrm{I} .52 \pm 0.8 \mathrm{I} /$ & $1.75 \pm 0.72 /$ \\
\hline$($ resin $[n=I \mid 7] /$ glass [ $n=38])(G B q)$ & $2.03 \pm 0.95$ & $2.00 \pm 0.0$ & $2.22 \pm 1.29$ & $1.95 \pm 0.83$ \\
\hline Single session whole liver & $1.2 \pm 0.55 /$ & & & \\
\hline (resin [n=II7]/glass [n=38]) (GBq) & $3.00 \pm 0.99$ & & & \\
\hline Unilobar (resin [n=II7]/ & $1.81 \pm 0.69 /$ & & & \\
\hline glass [n=38]) (GBq) & $1.95 \pm 0.72$ & & & \\
\hline Sequential bilobar (per lobe) & $1.62 \pm 0.89 /$ & & & \\
\hline (resin [n=I| 7] /glass [n=38]) (GBq) & $2.13 \pm 1.35$ & & & \\
\hline Repeated RE (resin [n=||7]/glass & $1.42 \pm 0.77 /$ & & & \\
\hline$[\mathrm{n}=38])(\mathrm{GBq})$ & $1.84 \pm 0.89$ & & & \\
\hline \multicolumn{5}{|l|}{ Device of primary RE (\%) } \\
\hline Resin & 87 (75.7) & $6(85.7)$ & $32(82.1)$ & $49(71.0)$ \\
\hline Glass & $28(24.3)$ & I (I4.3) & 7 (I7.9) & $20(29.0)$ \\
\hline Early stasis in resin-based RE & $13(14.9)$ & $0(0)$ & $6(18.8)$ & $7(14.3)$ \\
\hline \multicolumn{5}{|l|}{ Vessel embolization (\%) } \\
\hline No & $49(42.6)$ & $4(57.1)$ & $16(41.0)$ & $29(42.0)$ \\
\hline GDA & $37(32.2)$ & $3(42.9)$ & $12(30.8)$ & $22(31.9)$ \\
\hline GDX & $4(3.5)$ & $0(0)$ & $2(5.1)$ & $2(2.9)$ \\
\hline GDA + GDX & $25(21.7)$ & $0(0)$ & $9(23.1)$ & $16(23.2)$ \\
\hline \multicolumn{5}{|l|}{ TIPS (\%) } \\
\hline Yes & $4(3.5)$ & $0(0)$ & $2(5.1)$ & $2(2.9)$ \\
\hline No & III (96.5) & $0(0)$ & $37(94.9)$ & $67(97.1)$ \\
\hline Mean pulmonary shunt & $5.7 \pm 5.6$ & $3.7 \pm 4.7$ & $6.5 \pm 7.2$ & $5.5 \pm 4.6$ \\
\hline fraction (range) $(n=75)$ & $(0.1-35.0)$ & $(0.8-13.0)$ & $(1.0-35.0)$ & $(0.1-2 \mid .3)$ \\
\hline \multicolumn{5}{|l|}{ Extrahepatic progression after RE (\%) } \\
\hline Yes & II (9.6) & I (I4.3) & $2(5.1)$ & $8(11.6)$ \\
\hline No & $104(90.4)$ & $6(85.7)$ & $37(94.9)$ & $61(88.4)$ \\
\hline
\end{tabular}

Abbreviations: ALT, alanine-aminotransferase; AST, aspartate-aminotransferase; BCLC, Barcelona Clinic Liver Cancer; ECOG, Eastern Co-operative Oncology Group; GDA, gastroduodenal artery; GDX, right gastric artery; GGT, gamma glutamyl transferase; HCC, hepatocellular carcinoma; MELD, model for end-stage liver disease; RE, radioembolization; TACE, transarterial chemoembolization; TIPS, transjugular intrahepatic portosystemic shunt. 
Table 2 Laboratory and clinical toxicities according to CTCAE v. 4.0

\begin{tabular}{|c|c|c|c|c|c|c|c|c|c|c|c|c|c|c|c|c|c|c|}
\hline \multirow[t]{3}{*}{ Parameter } & \multicolumn{6}{|c|}{ Whole liver RE } & \multicolumn{6}{|c|}{ Sequential lobar RE* } & \multicolumn{6}{|c|}{ Unilobar RE } \\
\hline & \multicolumn{6}{|c|}{ Toxicity grade } & \multicolumn{6}{|c|}{ Toxicity grade } & \multicolumn{6}{|c|}{ Toxicity grade } \\
\hline & $\mathbf{N}$ & 0 & $\mathbf{I}$ & 2 & 3 & 4 & $\mathbf{N}$ & 0 & I & 2 & 3 & 4 & $\mathbf{N}$ & 0 & $\mathbf{I}$ & 2 & 3 & 4 \\
\hline \multicolumn{19}{|l|}{ Bilirubin } \\
\hline Baseline & 18 & 13 & 3 & 2 & 0 & 0 & 18 & 8 & 6 & 4 & 0 & 0 & 72 & $4 I$ & 17 & II & 3 & 0 \\
\hline I week & 14 & 9 & I & 4 & 0 & 0 & 15 & 9 & 4 & 0 & 2 & 0 & 66 & 39 & 14 & 11 & 2 & 0 \\
\hline I month & 8 & 5 & I & I & I & 0 & 11 & 5 & 0 & 3 & 3 & 0 & 41 & 30 & 4 & 3 & 4 & 0 \\
\hline 3 months & 10 & 6 & 2 & 0 & 2 & 0 & II & 3 & 2 & 3 & 2 & I & 36 & 17 & 8 & 9 & I & I \\
\hline \multicolumn{19}{|l|}{ ALT } \\
\hline Baseline & 18 & 6 & 9 & I & 2 & 0 & 19 & 2 & 13 & 3 & 0 & 0 & 73 & 30 & 35 & 7 & I & 0 \\
\hline I week & 14 & 4 & 7 & 2 & I & 0 & 15 & 0 & II & 4 & 0 & 0 & 68 & 20 & 38 & 4 & 6 & 0 \\
\hline I month & 8 & 5 & 2 & I & 0 & 0 & 13 & 2 & 9 & I & I & 0 & 42 & 19 & 19 & 2 & 2 & 0 \\
\hline 3 months & 10 & 7 & 2 & I & 0 & 0 & 11 & 1 & 8 & 2 & 0 & 0 & 37 & 15 & 15 & 3 & 4 & 0 \\
\hline \multicolumn{19}{|l|}{ AST } \\
\hline Baseline & 18 & 4 & 8 & 4 & 2 & 0 & 19 & 1 & II & 4 & 3 & 0 & 72 & 14 & 40 & 12 & 6 & 0 \\
\hline I week & 14 & 3 & 7 & 2 & 2 & 0 & 15 & 1 & 8 & 5 & I & 0 & 67 & 10 & 32 & 11 & 13 & I \\
\hline I month & 6 & 3 & 3 & 0 & 0 & 0 & 10 & 0 & 9 & I & 0 & 0 & 39 & 9 & 24 & 3 & 3 & 0 \\
\hline 3 months & 8 & 2 & 4 & I & 0 & I & 9 & 1 & 3 & 3 & 2 & 0 & 31 & 4 & 18 & 3 & 6 & 0 \\
\hline \multicolumn{19}{|l|}{ GGT } \\
\hline Baseline & 18 & 0 & 3 & 4 & 10 & I & 19 & 0 & I & 15 & 3 & 0 & 72 & 2 & 12 & 20 & 34 & 4 \\
\hline I week & 14 & 0 & 2 & 5 & 7 & 0 & 15 & 0 & I & II & 3 & 0 & 68 & 0 & 12 & 20 & 34 & 2 \\
\hline I month & 9 & 0 & 2 & 3 & 4 & 0 & II & 0 & 2 & 8 & I & 0 & 42 & 0 & 9 & 12 & 20 & I \\
\hline 3 months & 9 & 0 & 2 & 3 & 4 & 0 & 10 & 0 & I & 8 & I & 0 & 36 & 0 & 5 & 6 & 23 & 2 \\
\hline \multicolumn{19}{|l|}{ Ascites } \\
\hline Baseline & 18 & 14 & 3 & I & 0 & 0 & 19 & 14 & 5 & 0 & 0 & 0 & 73 & 54 & 17 & 2 & 0 & 0 \\
\hline After RE & 17 & 6 & 8 & 3 & 0 & 0 & 17 & 2 & 12 & 3 & 0 & 0 & 66 & 36 & 23 & 7 & 0 & 0 \\
\hline
\end{tabular}

Note: *After completed whole liver treatment.

Abbreviations: ALT, alanine aminotransferase; AST, aspartate aminotransferase; CTCAE, common terminology criteria for adverse events; GGT, gamma glutamyl transferase; RE, radioembolization.

\section{Tumor response and TTP}

Imaging response is summarized in Table 4. An objective response (ORR) rate (complete response + partial response [CR + PR]) of $43.3 \%$ and a disease control rate (DCR) $(\mathrm{CR}+\mathrm{PR}+$ stable disease $[\mathrm{SD}])$ of $83.3 \%$ were observed for the treated liver area. For the whole liver (including untreated tissue), ORR and DCR were $35.6 \%$ and $76.6 \%$, respectively. In patients undergoing repeated $R E(n=21)$, imaging follow-up was available in 16 cases (ORR and DCR $31.3 \%$ and $68.8 \%$, respectively).

Overall intrahepatic disease progression of the treated part of the liver was observed in $40 / 90$ patients $(44.4 \%)$ with imaging follow-up. Median TTHP treated $_{\text {was }} 4(0.9-45.4)$ months. Twenty-one out of 40 patients $(52.5 \%)$ with disease progression in the treated part of the liver showed new tumor manifestations in the treated liver lobe, $7 / 40(17.5 \%)$ showed growth of treated tumors and 12/40 (30\%) demonstrated both.

Progression in the untreated part was observed in $32 / 72$ patients $(44.4 \%)$ with a median TTHP $_{\text {untreated }}$ of 2 (0.9-45.6) months. Twenty out of 32 patients (62.5\%) showed new tumor manifestations in the untreated part of the liver, 5/32 (15.6\%) showed growth of existing tumor and $7 / 32(21.9 \%)$ showed both.

Table 3 Increase in laboratory toxicity levels after radioembolization (according to CTCAE v. 4.0)

\begin{tabular}{|c|c|c|c|c|c|c|c|c|c|}
\hline & \multicolumn{3}{|c|}{ Whole liver RE } & \multicolumn{3}{|c|}{ Sequential lobar RE* } & \multicolumn{3}{|c|}{ Unilobar RE } \\
\hline & $\begin{array}{l}\text { No } \\
\text { increase }\end{array}$ & $\begin{array}{l}\text { Temporary } \\
\text { increase }\end{array}$ & $\begin{array}{l}\text { Permanent } \\
\text { increase }\end{array}$ & $\begin{array}{l}\text { No } \\
\text { increase }\end{array}$ & $\begin{array}{l}\text { Temporary } \\
\text { increase }\end{array}$ & $\begin{array}{l}\text { Permanent } \\
\text { increase }\end{array}$ & $\begin{array}{l}\text { No } \\
\text { increase }\end{array}$ & $\begin{array}{l}\text { Temporary } \\
\text { increase }\end{array}$ & $\begin{array}{l}\text { Permanent } \\
\text { increase }\end{array}$ \\
\hline Bilirubin (\%) & $10(58.8)$ & $2(11.8)$ & $5(29.4)$ & $6(33.3)$ & I (5.6) & II (6I.I) & $49(68.0)$ & $2(2.8)$ & $21(29.2)$ \\
\hline ALT (\%) & $13(76.5)$ & 0 & $4(23.5)$ & $15(83.3)$ & $2(I I .1)$ & I (5.6) & $49(67.1)$ & $4(5.5)$ & $20(27.4)$ \\
\hline AST (\%) & $12(70.6)$ & $2(11.8)$ & $3(17.6)$ & $12(66.7)$ & $2(11.1)$ & $4(22.2)$ & $44(6 \mathrm{I} .1)$ & $6(8.3)$ & $22(30.6)$ \\
\hline GGT (\%) & 14 (82.3) & I (5.9) & $2(11.8)$ & 16 (88.9) & 0 & $2(I I . I)$ & $54(74.0)$ & I (I.4) & I8 (24.6I) \\
\hline
\end{tabular}

Note: *Increasing values are categorized as "permanent" if persisting at the last available follow-up.

Abbreviations: ALT, alanine aminotransferase; AST, aspartate-aminotransferase; CTCAE, common terminology criteria for adverse events; GGT, gamma glutamyl transferase; RE, radioembolization. 
Table 4 Best imaging response to radioembolization in patients with available imaging follow-up according to mRECIST-criteria on a per-patient basis after completion of a RE-cycle

\begin{tabular}{|c|c|c|}
\hline & $\begin{array}{l}\text { Treated } \\
\text { liver area }\end{array}$ & $\begin{array}{l}\text { Liver } \\
\text { overall }\end{array}$ \\
\hline \multicolumn{3}{|l|}{ Overall (\%) } \\
\hline Number of patients & 90 & 90 \\
\hline $\mathrm{CR}$ & $8(8.9)$ & $6(6.7)$ \\
\hline PR & 31 (34.4) & $26(28.9)$ \\
\hline SD & $36(40.0)$ & $37(4 I . I)$ \\
\hline PD & $15(16.7)$ & $21(23.3)$ \\
\hline \multicolumn{3}{|l|}{ Unilobar (\%) } \\
\hline Number of patients & 60 & 60 \\
\hline $\mathrm{CR}$ & $6(10.0)$ & $4(6.7)$ \\
\hline PR & $18(30.0)$ & $13(2 \mid .7)$ \\
\hline SD & $28(46.7)$ & $29(48.3)$ \\
\hline PD & $8(13.3)$ & $14(23.3)$ \\
\hline \multicolumn{3}{|c|}{ Whole liver single session (\%) } \\
\hline Number of patients & 14 & \\
\hline$C R$ & $7(50)$ & \\
\hline PR & $3(2 \mid .4)$ & \\
\hline SD & $4(28.6)$ & \\
\hline PD & $0(0)$ & \\
\hline \multicolumn{3}{|c|}{ Whole liver sequential (\%) } \\
\hline Number of patients & 16 & \\
\hline$C R$ & $2(12.5)$ & \\
\hline PR & $6(37.5)$ & \\
\hline SD & $5(3 \mid .3)$ & \\
\hline PD & $3(18.7)$ & \\
\hline \multicolumn{3}{|l|}{ Repeated RE (\%) } \\
\hline Number of patients & 16 & 16 \\
\hline $\mathrm{CR}$ & $0(0)$ & $0(0)$ \\
\hline PR & $5(3 \mid .3)$ & $2(12.5)$ \\
\hline SD & $6(37.5)$ & $9(56.3)$ \\
\hline PD & $5(3 \mid .3)$ & $5(3 \mid .3)$ \\
\hline
\end{tabular}

Abbreviations: $\mathrm{CR}$, complete response; $m R E C I S T$, modified response evaluation criteria in solid tumors; PD, progressive disease; PR, partial response; RE, radioembolization; SD, stable disease.

Eleven out of 90 patients (12.2\%) showed progression of extrahepatic disease during imaging follow-up (median TTEP 2.9 [1-43.7] months).

\section{Patient survival}

Thirty-day mortality rate was $2.6 \%$ (3/115 patients). A 65 -yearold patient (BCLC-C, ECOG [Eastern Co-operative Oncology Group] 2, tumor burden $>50 \%$, baseline bilirubin $3 \mathrm{mg} / \mathrm{dL}$ ) received resin-based unilobar treatment with $3.3 \mathrm{GBq}$ early in our institutional experience with $\mathrm{RE}$ and died 9 days after RE. A 79-year-old patient (BCLC-C, ECOG 1, tumor burden $25 \%-50 \%$, baseline bilirubin of $1.5 \mathrm{mg} / \mathrm{dL}$ ) with tumor invasion into the portal vein received unilobar treatment with $2 \mathrm{GBq}$ and died 21 days later. A third patient (71 years old) developed septicemia 2 days after RE and died 7 days later.
Median OS after first RE was 8.4 (0.3-82.8) months (BCLC-A: 52.8 months, BCLC-B: 12.4 months, BCLC-C: 6.1 months). Results of uni- and multivariate analyses of OS are summarized in Table 5. For the whole patient group, univariate analysis of OS showed significant interrelations with the ECOG status, liver tumor burden, BCLC status, Model for End-Stage Liver Disease (MELD) score, presence of ascites prior to RE, previous liver resection, baseline levels of bilirubin, AST, GGT and best overall response, TTP and repeated RE. On multivariate analysis, the ECOG status ( $p=0.001$, HR for ECOG $0 \mathrm{vs} \geq 1: 0.366$ ), the presence and extent of ascites prior to $\mathrm{RE}$ ( $p=0.029$, HR for no vs extensive ascites: 0.039 and minimal vs extensive ascites: 0.044 ) and best overall mRECIST response ( $p<0.0001$, HR for CR, PR and SD vs PD: $0.071,0.331$ and 1.135 , respectively) were identified as independent predictors of OS. There was a trend toward longer OS in patients without baseline bilirubin toxicity ( $p=0.052$, HR 0.543) (Figure 1).

Analysis of BCLC-B patients did not show any significant associations of patient-related factors and survival. In BCLC-C patients, univariate analysis showed significant interrelations of OS and patient age, liver tumor burden, Child-Pugh status, MELD score, presence of ascites prior to intervention, previous liver resection, previous application of sorafenib, baseline levels of bilirubin or GGT and repeated RE. On multivariate analysis, presence and extent of ascites prior to $\mathrm{RE}$ ( $p=0.008$, HR for no vs extensive ascites: 0.156 and minimal vs extensive ascites: 0.266 ), baseline GGT $(p=0.011$, HR for $\leq 1$ vs $>1: 0.288)$ and Child-Pugh score $(p<0.031$, HR for Child-Pugh A vs B: 0.412) were identified as independent predictors of OS.

\section{Discussion}

Although TACE or sorafenib therapy can improve survival in HCC patients, ${ }^{2,12}$ tolerability (eg, toxicities in the majority of patients with sorafenib) ${ }^{13}$ and applicability (eg, TACE in patients with portal vein thrombosis) remain problematic. Liver failure is the cause of death in about $90 \%$ of advanced HCC, even in the presence of extrahepatic metastases, which underlines the importance of liver-directed therapy. ${ }^{2,25-29} \mathrm{RE}$ has been investigated in several small studies with promising results. However, until now, only a limited number of studies, including $>100$ patients has been published. ${ }^{30-32}$

Clinically, disease control and ORRs of RE for HCC are ranging from $77 \%$ to $90 \% \%^{30,33,34}$ to $40 \%-57 \%,{ }^{30,32}$ respectively. Considering the dismal prognosis, especially of advanced stage $\mathrm{HCC}^{35}$ with otherwise only limited therapeutic options, 
Table 5 Results of univariate analysis of overall survival after first radioembolization

\begin{tabular}{|c|c|c|c|c|c|c|}
\hline \multirow[t]{2}{*}{ Parameter } & \multicolumn{2}{|l|}{ Overall } & \multicolumn{2}{|l|}{ BCLC-B } & \multicolumn{2}{|l|}{ BCLC-C } \\
\hline & $\begin{array}{l}\text { Median survival } \\
\text { (months) }(95 \% \mathrm{Cl})\end{array}$ & $\begin{array}{l}\text { Univariate } \\
\text { p-value }\end{array}$ & $\begin{array}{l}\text { Median survival } \\
\text { (months) }(95 \% \mathrm{Cl})\end{array}$ & $\begin{array}{l}\text { Univariate } \\
p \text {-value }\end{array}$ & $\begin{array}{l}\text { Median survival } \\
\text { (months) }(95 \% \mathrm{Cl})\end{array}$ & $\begin{array}{l}\text { Univariate } \\
p \text {-value }\end{array}$ \\
\hline \multicolumn{7}{|l|}{ Gender } \\
\hline Female & $7.7(5.8 ; 9.7)$ & 0.710 & $9.2(1.2 ; 172)$ & 0.508 & $4.3(2.8 ; 5.8)$ & 0.828 \\
\hline Male & $8.4(6.5 ; 10.3)$ & & | 3.4 (7.2; 19.7) & & $6.5(3.6 ; 9.3)$ & \\
\hline Mean age (range) & & 0.325 & & 0.944 & & 0.022 \\
\hline \multicolumn{7}{|l|}{ (at time of RE) } \\
\hline Time between first & & 0.210 & & 0.643 & & 0.748 \\
\hline \multicolumn{7}{|l|}{ diagnosis of HCC } \\
\hline \multicolumn{7}{|l|}{ and RE (n=99) } \\
\hline \multicolumn{7}{|l|}{ ECOG status $(n=84)$} \\
\hline 0 & $13.6(7.5 ; 19.7)$ & 0.004 & $9.0(0 ; 19.8)$ & 0.935 & $12.3(3.0 ; 21.5)$ & 0.103 \\
\hline$\geq 1$ & $6.5(4.2 ; 8.7)$ & & $9.8(5.8 ; 13.8)$ & & $6.0(3.9 ; 8.1)$ & \\
\hline \multicolumn{7}{|c|}{ Estimated relative liver tumour burden } \\
\hline$\leq 25 \%$ & I3.I (8.0; I8.2) & 0.0002 & $14.2(8.2 ; 20.3)$ & 0.532 & $10.3(1.5 ; 13.2)$ & 0.014 \\
\hline$>25 \%$ & $6.5(4.6 ; 8.3)$ & & $8.2(6.8 ; 11.2)$ & & $4.9(3.1 ; 6.6)$ & \\
\hline \multicolumn{7}{|c|}{ Pattern of tumour manifestation } \\
\hline Solitary & $9.0(0 ; 25.5)$ & 0.187 & I $3.6(4.9 ; 22.4)$ & 0.643 & $3.9(2.2 ; 5.6)$ & 0.790 \\
\hline Oligofocal & $13.1(7.4 ; 15.5)$ & & II.5 (I.9; 21.0) & & I3.I $(5.9 ; 20.3)$ & \\
\hline Multifocal & $8.0(6.7 ; 9.4)$ & & $9.8(0.7 ; 18.9)$ & & $6.1(2.6 ; 9.6)$ & \\
\hline Diffuse & $4.2(0 ; 14.1)$ & & & & $4.2(0 ; \mid 4.1)$ & \\
\hline \multicolumn{7}{|c|}{ Distribution of metastases } \\
\hline Unilobar right & I3.I (7.5; 18.7) & 0.127 & $16.2(13.2 ; 19.3)$ & 0.928 & $4.9(0 ; 14.6)$ & 0.704 \\
\hline Unilobar left & $5.7(2.0 ; 9.4)$ & & $57(4.8 ; 6.6)$ & & $8.0(0 ; 16.5)$ & \\
\hline Bilobar & $8.1(6.5 ; 9.6)$ & & $9.2(3.2 ; 15.2)$ & & $6.0(3.1 ; 8.8)$ & \\
\hline \multicolumn{7}{|l|}{ Liver cirrhosis } \\
\hline Yes & 8.1 (6.I; 10.I) & 0.357 & $36.3(0 ; 59.8)$ & 0.085 & $8.0(2.8 ; 13.2)$ & 0.991 \\
\hline No & $9.0(7.4 ; 10.5)$ & & $9.8(3.3 ; 16.3)$ & & $6.0(3.9 ; 8.1)$ & \\
\hline \multicolumn{7}{|l|}{ Cause of cirrhosis } \\
\hline Hepatitis & $8.4(6.9 ; 9.8)$ & 0.682 & I3.6 (0.7; 26.6) & 0.816 & $8.0(6.5 ; 9.4)$ & 0.258 \\
\hline Alcohol & $6.2(4.6 ; 7.7)$ & & $9.8(0 ; 19.6)$ & & $3.1(1.2 ; 5.0)$ & \\
\hline \multicolumn{7}{|l|}{ Child-Pugh status } \\
\hline$A$ & $8.4(7.2 ; 9.6)$ & 0.110 & & & $7.1(4.5 ; 9.6)$ & 0.003 \\
\hline B & $1.7(1.3 ; 2.1)$ & & & & I.5 (I.I; I.9) & \\
\hline \multicolumn{7}{|l|}{$\mathrm{BCLC}$} \\
\hline A & $52.8(0 ; 109.7)$ & 0.0005 & & & & \\
\hline B & $12.4(4.9 ; 18.0)$ & & & & & \\
\hline C & $6.1(3.2 ; 9.0)$ & & & & & \\
\hline Mean MELD score & & $<0.0001$ & & 0.498 & & 0.005 \\
\hline \multicolumn{7}{|l|}{ Ascites prior to RE } \\
\hline No & $9.2(7.2 ; 11.2)$ & $<0.0001$ & II.5 (6.2; 16.7) & 0.876 & $8.4(6.7 ; 10.1)$ & 0.004 \\
\hline Minimal & $6.0(4.2 ; 7.8)$ & & 16.2 & & $4.5(2.3 ; 6.7)$ & \\
\hline Extensive & $2.1(1.4 ; 2.7)$ & & & & $2.1(1.4 ; 2.7)$ & \\
\hline Extrahepatic diseas & & & & & & \\
\hline Yes & $9.0(6.6 ; 11.4)$ & 0.772 & & & $7.1(4.2 ; 10.0)$ & 0.111 \\
\hline No & $7.7(5.4 ; 10.0)$ & & & & $3.6(0 ; 7.4)$ & \\
\hline Vascular invasion & & & & & & \\
\hline Yes & $6.0(1.6 ; 10.4)$ & 0.058 & & & $6(1.6 ; 10.4)$ & 0.659 \\
\hline No & $8.4(7.1 ; 9.6)$ & & & & $4.3(0.8 ; 7.7)$ & \\
\hline Previous liver resec & & & & & & \\
\hline Yes & I3.6 (7.3; 20.0) & 0.012 & I3.6 (3.8; 23.5) & 0.606 & $8.4(3.9 ; 8.0)$ & 0.033 \\
\hline No & $7.7(6.1 ; 6.9)$ & & II.5 (3.6; 19.3) & & $6.0(3.9 ; 8.0)$ & \\
\hline Previous application & orafenib & & & & & \\
\hline Yes & $12.3(7.4 ; 17.1)$ & 0.122 & | 3.4 (7.2; 19.7) & 0.929 & $8.4(2.7 ; 14.1)$ & 0.040 \\
\hline No & $8.1(6.2 ; 10.0)$ & & $9.8(2.7 ; 16.9)$ & & $5.2(2.9 ; 7.5)$ & \\
\hline Previous TACE & & & & & & \\
\hline Yes & $8.4(6.1 ; 10.7)$ & 0.939 & $9.8(5.7 ;$ I3.9) & 0.435 & $6.0(3.7 ; 8.3)$ & 0.930 \\
\hline No & $8.2(7.4 ; 9.1)$ & & I5.5 (5.8; 25.2) & & $6.5(2.8 ; 9.8)$ & \\
\hline
\end{tabular}


Table 5 (Continued)

\begin{tabular}{|c|c|c|c|c|c|c|}
\hline \multirow[t]{2}{*}{ Parameter } & \multicolumn{2}{|l|}{ Overall } & \multicolumn{2}{|l|}{ BCLC-B } & \multicolumn{2}{|l|}{ BCLC-C } \\
\hline & $\begin{array}{l}\text { Median survival } \\
\text { (months) }(95 \% \mathrm{Cl})\end{array}$ & $\begin{array}{l}\text { Univariate } \\
\text { p-value }\end{array}$ & $\begin{array}{l}\text { Median survival } \\
\text { (months) }(95 \% \mathrm{Cl})\end{array}$ & $\begin{array}{l}\text { Univariate } \\
p \text {-value }\end{array}$ & $\begin{array}{l}\text { Median survival } \\
\text { (months) }(95 \% \mathrm{Cl})\end{array}$ & $\begin{array}{l}\text { Univariate } \\
\text { p-value }\end{array}$ \\
\hline \multicolumn{7}{|c|}{ Baseline bilirubin toxicity grade } \\
\hline 0 & $10.6(7.2 ; 14.0)$ & 0.001 & | $3.6(9.1 ; \mid 8.1)$ & 0.502 & $8.4(6.9 ; 9.9)$ & 0.022 \\
\hline$\geq 1$ & $4.5(2.6 ; 6.4)$ & & $5.7(4.6 ; 6.8)$ & & $3.6(1.5 ; 5.7)$ & \\
\hline \multicolumn{7}{|c|}{ Baseline ALT toxicity grade } \\
\hline 0 & $9.3(4,5 ; 14.0)$ & 0.201 & $15.5(8.4 ; 22.6)$ & 0.071 & $8.0(5.0 ; 10.9)$ & 0.942 \\
\hline$\geq 1$ & $4.5(4.9 ; 10.0)$ & & $9.0(6.6 ; 11.3)$ & & $4.9(1.9 ; 7.9)$ & \\
\hline \multicolumn{7}{|c|}{ Baseline AST toxicity grade } \\
\hline 0 & $18.9(\mid 3.8 ; 24.0)$ & 0.002 & $21.2(8.2 ; 34.2)$ & 0.124 & $12.3(9.2 ; 15.4)$ & 0.129 \\
\hline$\geq 1$ & $7.5(5.5 ; 9.4)$ & & $9.2(6.8 ; 1.6)$ & & $5.2(3.4 ; 7.0)$ & \\
\hline \multicolumn{7}{|c|}{ Baseline GGT toxicity grade } \\
\hline$\leq 1$ & $18.9(6.9 ; 30.9)$ & 0.001 & $15.5(10.7 ; 20.3)$ & 0.451 & $18.9(0.2 ; 37.6)$ & 0.033 \\
\hline$>1$ & $7.7(6.0 ; 9.5)$ & & $9.2(5.4 ; 13.0)$ & & $6.0(3.9 ; 8.0)$ & \\
\hline \multicolumn{7}{|c|}{ Mean radioembolization activity $(\mathrm{n}=156)$} \\
\hline Administered & & 0.111 & & 0.290 & & 0.264 \\
\hline \multicolumn{7}{|l|}{ Device of primary RE } \\
\hline Resin & $8.3(6.9 ; 9.6)$ & 0.303 & $9.8(2.7 ; 16.9)$ & 0.348 & $6.0(3.7 ; 8.3)$ & 0.580 \\
\hline Glass & $9.9(5.6 ; 14.2)$ & & II.5 $(2.7 ; 20.3)$ & & $8.0(0.4 ; 15.5)$ & \\
\hline \multicolumn{7}{|l|}{ TIPS } \\
\hline Yes & $5.7(0 ; 16.9)$ & 0.710 & II.5 (5.I; I7.8) & 0.724 & $6.1(3.3 ; 8.9)$ & 0.985 \\
\hline No & $8.4(7.0 ; 9.7)$ & & 5.7 & & 2.7 & \\
\hline \multicolumn{7}{|c|}{ Extrahepatic progression after RE } \\
\hline Yes & $8.4(7.1 ; 9.7)$ & 0.140 & 4.6 & 0.699 & $4.3(1.7 ; 6.9)$ & 0.234 \\
\hline No & $6.1(1.8 ; 10.4)$ & & II.5 (5.I; I7.8) & & $6.5(3.5 ; 9.4)$ & \\
\hline \multicolumn{7}{|c|}{ Best overall hepatic response } \\
\hline CR & $18.9(0 ; 61.7)$ & 0.002 & & 0.188 & $18.9(18.4 ; 19.4)$ & 0.131 \\
\hline PR & $14.2(8.8 ; 19.6)$ & & $16.2(8.0 ; 24.4)$ & & $8.4(0 ; 17.1)$ & \\
\hline SD & $7.7(6.3 ; 9.1)$ & & $7.7(5.8 ; 9.7)$ & & $7.1(3.7 ; 10.5)$ & \\
\hline PD & $8.3(4.9 ; 11.7)$ & & $15.5(0 ; 34.8)$ & & $6.1(0.1 ; 12.1)$ & \\
\hline $\begin{array}{l}\text { Time to progression, } \\
\text { treated area }\end{array}$ & & 0.005 & & 0.628 & & 0.104 \\
\hline $\begin{array}{l}\text { Time to progression, } \\
\text { non-treated area }\end{array}$ & & 0.096 & & & & 0.243 \\
\hline \multicolumn{7}{|l|}{ Repeated RE } \\
\hline Yes & $16.2(3.8 ; 28.7)$ & 0.033 & II.5 (0; 23.6) & 0.697 & I7.| (2.5; 31.8$)$ & 0.002 \\
\hline No & $7.4(5.5 ; 9.4)$ & & $9.8(4.0 ; 15.6)$ & & $5.2(3.4 ; 7.0)$ & \\
\hline
\end{tabular}

Abbreviations: ALT, alanine aminotransferase; AST, aspartate aminotransferase; BCLC, Barcelona Clinic Liver Cancer; CR, complete response; ECOG, Eastern Cooperative Oncology Group; GGT, gamma glutamyl transferase; HCC, hepatocellular carcinoma; MELD, model for end-stage liver disease; PD, progressive disease; PR, partial response; RE, radioembolization; SD, stable disease; TACE, transarterial chemoembolization; TIPS, transjugular intrahepatic portosystemic shunt.

these are noteworthy treatment effects, comparable with response rates of TACE. ${ }^{36}$ TACE, however, is relatively contraindicated in patients with portal vein occlusion, limiting its application in this subgroup of patients. ${ }^{37}$ In addition, several TACE treatment sessions are usually necessary for successful therapy.

Early progression after RE primarily seems to depend on baseline characteristics like tumor distribution and extent rather than on procedural factors. ${ }^{33}$ This was also true in our patients. Since a higher percentage of patients in our study suffered from intermediate and advanced stage HCC compared with previous studies $(95.7 \%$ vs $80 \%)$, patients in our cohort showed an overall shorter median TTP (4 months vs
7.9-10 months).$^{30,32}$ Hepatic progression in general may arise from an increase in known tumor masses or new manifestations in treated liver areas. In unilobar therapy, untreated contralateral disease may progress or new manifestations may become detectable. In this respect, it has been suggested that disease progression after RE of HCC is associated with new tumor manifestations rather than growth of treated tumor. ${ }^{38-40}$ This is corroborated by comparable rates of disease progression in treated and untreated liver lobes with progression only developing later in treated liver in our cohort. Development of new tumor nodules was associated with progression in $82.5 \%$ of treated and $84.4 \%$ of untreated liver. While, especially small tumor nodules $(<3 \mathrm{~cm})$ show a high rate of histological 

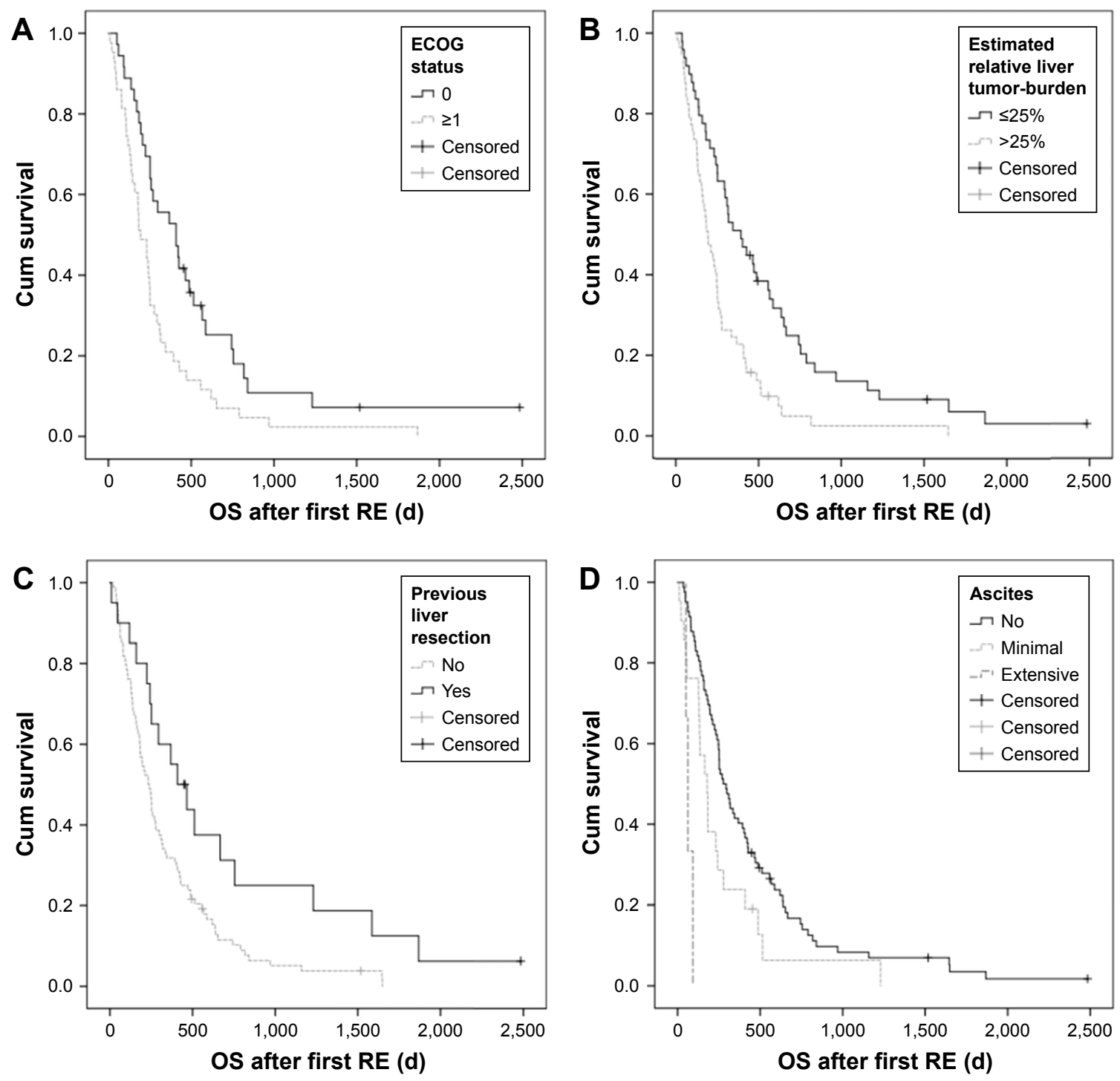

Figure I Kaplan-Meier curves.

Notes: (A) OS by ECOG status (broken line: ECOG >0, continuous line: ECOG 0); (B) OS by estimated relative liver tumor burden (broken line: $\geq 25 \%$ of liver volume, continuous line: $<25 \%$ ); (C) OS by previous surgical liver tumor resection (broken line: no resection, continuous line: previous resection); (D) OS by presence of ascites prior to RE (dark gray dashed line: extensive ascites, light gray broken line: minimal ascites continuous line: no ascites).

Abbreviations: ECOG, Eastern Co-operative Oncology Group; OS, overall survival; RE, radioembolization.

complete tumor response after RE $(89 \%),{ }^{41}$ microscopic tumor manifestations may be insufficiently treated due to lack of neoangiogenic vessels and may, therefore, give rise to post RE disease progression. ${ }^{40}$

Extrahepatic disease was already present in $35 \%$ of our patients at the time of RE, with only $12 \%$ showing extrahepatic progression on follow-up. Moreover, as liver failure is known to be the cause of death in the majority of patients, ${ }^{2,25-29}$ extrahepatic disease does not seem to be a contraindication for RE.

The importance of liver-directed treatment is reflected in the fact that OS can be considerably increased by implementing RE into the treatment algorithm. Patients with untreated
HCC have a median survival of only 9 months after initial diagnosis (BCLC-A: 25 months, B: 10 months, C: 7 months, D: 6 months). ${ }^{35}$ As RE was often performed rather late in the sequence of treatment options in our cohort, patients had already survived longer after initial diagnosis than they would have without any treatment (time from diagnosis to RE: BCLC A: 26.4 months, B: 17.3 months, C: 12 months). The added value of $\mathrm{RE}$ is reflected in considerably longer cumulative $\mathrm{OS}$ after initial diagnosis that amounted to a total of 77 months in BCLC-A, 29.7 months in BCLC-B and 18.1 months in BCLC-C in our patients. This is particularly interesting, since the added value of sorafenib in advanced stage HCC is a prolongation of mean OS of only 6 weeks. ${ }^{12-14}$ 
Patients receiving either resin- or glass-based RE are reported to have a median OS of 13-16 months after treatment (BCLC-A: 24.4-26.9 months, B: 16.9-17.2 months, C: 6.0-10.0 months). ${ }^{30-32}$ Patients in our cohort showed a shorter OS of 8.4 months, which was mainly influenced by the high rate of advanced stage $\mathrm{HCC}$ (median OS 6.1 months). Our patient cohort is comparable with the recently published prospective randomized controlled SARAH trial as far as a high percentage of BCLC-C patients was concerned. A slightly longer median OS in the SARAH trial (9.9 months vs 8.4 months) may be explained by the fact that we included patients with an ECOG status of 2 in the first years of our experience with RE over 10 years ago (about 19\% of the patient cohort) that were excluded in the SARAH trail. According to current inclusion criteria for RE, patients with an ECOG status of 2 would no longer be treated by RE. Compared with the SIRveNIB trail that included less BCLC-C patients (35\% vs 60\%) and also did not include ECOG 2 patients, OS was also longer in the SIRveNIB trial (11.3 months vs 8.4 months). ${ }^{42,43}$ Of note, median OS in the small group of patients with BCLC stage A in our cohort was considerably higher compared with previous reports (median OS 50.6 months). High tumor response rates do not generally translate into longer OS: while response rates are comparable for HCC treated with RE and TACE, OS is known to be significantly longer after RE. ${ }^{36}$ This can be due to different tumor and patient characteristics. In our patients, especially a low ECOG status and the absence of ascites prior to RE were predictive of longer OS. Furthermore, already identified prognostic factors include patient age, gender, presence of portal hypertension, extend and distribution of tumor burden, several laboratory values and extrahepatic disease. ${ }^{31,32}$ Considering these factors, lower OS in our study may be explained by the fact that our patients showed adverse prognostic parameters in a larger percentage than in previously published reports. Among other factors, patients had a higher ECOG status (ECOG 0: 45.2\% vs 54\%-56\% in previous reports), a higher tumor burden ( $\geq 25 \%$ : $53.1 \%$ vs $23.4 \%$ or $>5$ tumor nodules: $67.8 \%$ vs $38.6 \%$ ) and a higher incidence of extrahepatic disease (37.4\% vs 9.2\%-16\%). ${ }^{31,32}$

Especially in BCLC-C patients, adequate patient selection is imperative. Patients with complete portal vein thrombosis, who are typically no candidates for TACE,${ }^{44}$ have a dismal prognosis (median OS 2-4 months with best supportive care, 3-6 months after RE). ${ }^{15,17,34,45}$ We also found a median OS of 6.0 months in patients presenting with macroscopic vascular invasion. However, selected BCLC-C patients with a low tumor burden, absence of ascites, low baseline
GGT levels and Child-Pugh status A showed an OS of up to 18.9 months after RE, which is even above the previously reported median OS of intermediate stage patients.

There is ongoing debate on how to deal with recurrent progression after successful RE, especially as impaired hepatic function has to be taken into account when considering repeated liver-directed treatment. We offered repeated $\mathrm{RE}$ to patients with preserved liver function, ${ }^{21}$ developing recurrent disease progression after initial RE. We found that tumor control could be achieved in nearly $70 \%(11 / 16)$ of repeated REs with a significantly longer OS in patients receiving repeat RE. Although disease progression can be stopped in a substantial percentage of patients receiving repeat $\mathrm{RE}$, the fact that liver function had to be preserved to be considered for repeat RE certainly also influenced OS. However, appropriately selected BCLC-C patients seem to benefit from repeat RE (OS 17.1 vs 5.2 months). Further systematic investigation into adequate patient selection for repeated $\mathrm{RE}$, therefore, seems to be warranted.

Positive treatment effects are usually associated with a certain degree of adverse events. This holds especially true in advanced stage HCC patients treated with sorafenib who experience adverse events in up to $80 \%$, often warranting dose reduction, which, in turn, may have a detrimental effect on treatment efficacy. ${ }^{12-14}$ By comparison, RE is associated with a rather low toxicity profile in appropriately selected patients. ${ }^{46,47}$ Severe adverse events $\geq$ grade 3 (eg, gastroduodenal ulceration) occur in $<5 \%$ of procedures. ${ }^{48,49}$ We observed grade 2 gastroduodenal ulceration in $2.6 \%$ of cases although the gastroduodenal artery was coil-embolized. Additional vessels not identified on angiography and MAA test injection (eg, newly formed collaterals after coilembolization of the gastroduodenal artery) may have been the cause of extrahepatic non-target embolization in these cases. Early stasis during RE, a risk factor for gastroduodenal ulceration, ${ }^{50}$ is rare in $\mathrm{HCC}$ treatment ${ }^{51}$ and was only observed in 1 patient presenting with ulceration in our cohort.

Special care must be taken in cirrhotic patients, due to a reduced functional liver reserve and an increased risk of liver failure or RE-induced liver disease (REILD). ${ }^{52,53} \mathrm{We}$ found no or only minor biochemical toxicities in the majority of cases. Grade $\geq 3$ bilirubin toxicities within 3 months after RE (an indicator of REILD) occurred in $8.3 \%$ of patients, which is in agreement with reported values of $6 \%-14 \% .^{31,32}$ Development or worsening of ascites, another hallmark of REILD, occurred in $21.7 \%$, but was $\leq$ grade 2 in all cases. Sequential lobar therapy is thought to be better tolerated than whole liver treatment. ${ }^{54}$ However, we observed a higher rate 
of bilirubin toxicities in sequential bilobar compared with unilobar or simultaneous bilobar treatment, which may be due a higher cumulative administered dose in sequential treatment (3.2 GBq vs 1.5-1.8 GBq).

The results of our analysis are limited by the retrospective character of our study with inherent methodological problems. Several parameters, for example, the ECOG status or the exact cause of death could not be reconstructed in many patients, especially in those treated before systematic electronic archiving was implemented at our institution. Baseline alpha-fetoprotein (AFP) values were only available in a minority of patients and were therefore not included into the analysis. As decisions to perform RE were reached in interdisciplinary tumor boards based on the individual patient history, the patient cohort was rather heterogenic, especially concerning therapy regimes prior to and after RE. Although data of retrospectively published cohorts generally seem to be in line with each other, prospectively conducted, preferably randomized controlled trials are imperative for implementation of RE into HCC therapy algorithms.

\section{Conclusion}

The results of our study add to the growing literature on RE of HCC, demonstrating that RE is safe and effective in carefully selected patients. It stops progression of HCC in a large percentage of patients even in advanced stages. Progression after RE is associated rather with the development of new HCC nodules than the growth of treated tumors. Especially patients with a low baseline ECOG status without ascites may benefit from treatment. In addition, patients with advanced HCC should be carefully selected according to the presence of ascites, baseline GGT and Child-Pugh class. Repeated RE can prolong survival in BCLC-C patients with adequate liver function who demonstrate recurrent progression after first RE.

\section{Acknowledgment}

Jennifer Nadal (Institute for Medical Biometry, Informatics and Epidemiology, University of Bonn) provided statistical advice.

\section{Disclosure}

$\mathrm{CM}$ is a consultant for SIRTEX Medical, PharmaCept and GoreMedical. The authors report no other conflicts of interest in this work.

\section{References}

1. Bosch FX, Ribes J, Díaz M, Cléries R. Primary liver cancer: worldwide incidence and trends. Gastroenterology. 2004;127(5 Suppl 1):S5-S16.

2. Llovet JM, Ducreux M. European Association For The Study Of The Liver; European Organization For Research And Treatment Of Cancer. EASL-EORTC clinical practice guidelines: management of hepatocellular carcinoma. $J$ Hepatol. 2012;56(4):908-943.
3. Lencioni R, Cioni D, Crocetti L, et al. Early-stage hepatocellular carcinoma in patients with cirrhosis: long-term results of percutaneous imageguided radiofrequency ablation. Radiology. 2005;234(3):961-967.

4. Bruix J, Sherman M; Practice Guidelines Committee, American Association for the Study of Liver Diseases. Management of hepatocellular carcinoma. Hepatology. 2005;42(5):1208-1236.

5. Elnekave E, Erinjeri JP, Brown KT, et al. Long-term outcomes comparing surgery to embolization-ablation for treatment of solitary HCC $<7$ cm. Ann Surg Oncol. 2013;20(9):2881-2886.

6. Zhao M, Wang JP, Pan CC, et al. CT-guided radiofrequency ablation after with transarterial chemoembolization in treating unresectable hepatocellular carcinoma with long overall survivalimprovement. Eur J Radiol. 2012;81(10):2717-2725.

7. Veltri A, Moretto P, Doriguzzi A, Pagano E, Carrara G, Gandini G. Radiofrequency thermal ablation (RFA) after transarterial chemoembolization (TACE) as a combined therapy for unresectable non-early hepatocellular carcinoma (HCC). Eur Radiol. 2006;16(3):661-669.

8. Kagawa T, Koizumi J, Kojima S, et al; Tokai RFA Study Group. Transcatheter arterial chemoembolization plus radiofrequency ablation therapy for early stage hepatocellular carcinoma: comparison with surgical resection. Cancer. 2010;116(15):3638-3644.

9. Malagari K, Pomoni M, Kelekis A, et al. Prospective randomized comparison of chemoembolization with doxorubicin-eluting beads and bland embolization with BeadBlock for hepatocellular carcinoma. Cardiovasc Intervent Radiol. 2010;33(3):541-551.

10. Golfieri R, Giampalma E, Renzulli M, et al. Randomized controlled trial of doxorubicin-eluting beads vs conventional chemoembolisation for hepatocellular carcinoma. Br J Cancer. 2014;111(2):255-264.

11. Llovet JM, Real MI, Montaña X, et al. Arterial embolisation or chemoembolisation vs symptomatic treatment in patients with unresectable hepatocellular carcinoma: a randomized controlled trial. Lancet. 2002;359(9319):1734-1739.

12. Llovet JM, Ricci S, Mazzaferro V, et al; SHARP Investigators Study Group. Sorafenib in advanced hepatocellular carcinoma. $N$ Engl J Med. 2008;359(4):378-390.

13. Cheng AL, Kang YK, Chen Z, et al. Efficacy and safety of sorafenib in patients in the Asia-Pacific region with advanced hepatocellular carcinoma: a phase III randomized, double-blind, placebo-controlled trial. Lancet Oncol. 2009;10(1):25-34.

14. Li Y, Gao ZH, Qu XJ. The adverse effects of sorafenib in patients with advanced cancers. Basic Clin Pharmacol Toxicol. 2015;116(3): 216-221.

15. Llovet JM, Bustamante J, Castells A, et al. Natural history of untreated nonsurgical hepatocellular carcinoma: rationale for the design and evaluation of therapeutic trials. Hepatology. 1999;29(1):62-67.

16. Minagawa M, Makuuchi M. Treatment of hepatocellular carcinoma accompanied by portal vein tumor thrombus. World J Gastroenterol. 2006;12(47):7561-7567.

17. Schöniger-Hekele M, Müller C, Kutilek M, Oesterreicher C, Ferenci P, Gangl A. Hepatocellular carcinoma in Central Europe: prognostic features and survival. Gut. 2001;48(1):103-109.

18. Salem R, Mazzaferro V, Sangro B. Yttrium 90 radioembolization for the treatment of hepatocellular carcinoma: biological lessons, current challenges, and clinical perspectives. Hepatology. 2013;58(6): 2188-2197.

19. Salem R, Gordon AC, Mouli S, et al. Y90 Radioembolization significantly prolongs time to progression compared with chemoembolization in patients with hepatocellular carcinoma. Gastroenterology. 2016; 151(6):1155-1163.e2.

20. Salem R, Thurston KG. Radioembolization with 90Yttrium microspheres: a state-of-the-art brachytherapy treatment for primary and secondary liver malignancies. Part 1: technical and methodologic considerations. J Vasc Interv Radiol. 2006;17(8):1251-1278.

21. Kennedy A, Nag S, Salem R, et al. Recommendations for radioembolization of hepatic malignancies using yttrium-90 microsphere brachytherapy: a consensus panel report from the radioembolization brachytherapy oncology consortium. Int J Radiat Oncol Biol Phys. 2007; 68(1):13-23. 
22. European Association For The Study Of The Liver; European Organization For Research And Treatment Of Cancer. EASL-EORTC clinical practice guidelines: management of hepatocellular carcinoma. J Hepatol. 2012;56(4):908-943.

23. Mahnken AH, Spreafico C, Maleux G, Helmberger T, Jakobs TF. Standards of practice in transarterial radioembolization. Cardiovasc Intervent Radiol. 2013;36(3):613-622.

24. Lencioni R, Llovet JM. Modified RECIST (mRECIST) assessment for hepatocellular carcinoma. Semin Liver Dis. 2010;30(1):52-60.

25. Raoul JL, Gilabert M, Piana G. How to define transarterial chemoembolization failure or refractoriness: a European perspective. Liver Cancer. 2014;3(2):119-124.

26. Peck-Radosavljevic M. Drug therapy for advanced-stage liver cancer. Liver Cancer. 2014;3(2):125-131.

27. Kudo M. Biomarkers and personalized sorafenib therapy. Liver Cancer. 2014;3(3-4):399-404.

28. Forner A, Gilabert M, Bruix J, Raoul JL. Treatment of intermediate-stage hepatocellular carcinoma. Nat Rev Clin Oncol. 2014;11(9):525-535.

29. Uka K, Aikata H, Takaki S, et al. Clinical features and prognosis of patients with extrahepatic metastases from hepatocellular carcinoma. World J Gastroenterol. 2007;13(3):414-420.

30. Hilgard P, Hamami M, Fouly AE, et al. Radioembolization with yttrium-90 glass microspheres in hepatocellular carcinoma: European experience on safety and long-term survival. Hepatology. 2010;52(5): 1741-1749.

31. Sangro B, Carpanese L, Cianni R, et al; European Network on Radioembolization with Yttrium-90 Resin Microspheres (ENRY). Survival after yttrium-90 resin microsphere radioembolization of hepatocellular carcinoma across Barcelona clinic liver cancer stages: a European evaluation. Hepatology. 2011;54(3):868-878.

32. Salem R, Lewandowski RJ, Mulcahy MF, et al. Radioembolization for hepatocellular carcinoma using Yttrium-90 microspheres: a comprehensive report of long-term outcomes. Gastroenterology. 2010; 138(1):52-64.

33. Iñarrairaegui $M$, Martinez-Cuesta A, Rodríguez M, et al. Analysis of prognostic factors after yttrium-90 radioembolization of advanced hepatocellular carcinoma. Int J Radiat Oncol Biol Phys. 2010;77(5): 1441-1448.

34. Kulik LM, Carr BI, Mulcahy MF, et al. Safety and efficacy of $90 \mathrm{Y}$ radiotherapy for hepatocellular carcinoma with and without portal vein thrombosis. Hepatology. 2008;47(1):71-81.

35. Giannini EG, Farinati F, Ciccarese F, et al; Italian Liver Cancer (ITA LI.CA) group. Prognosis of untreated hepatocellular carcinoma. Hepatology. 2015;61(1):184-190.

36. Zhang Y, Li Y, Ji H, Zhao X, Lu H. Transarterial Y90 radioembolization vs chemoembolization for patients with hepatocellular carcinoma: a meta-analysis. Biosci Trends. 2015;9(5):289-298.

37. Lau WY, Sangro B, Chen PJ, et al. Treatment for hepatocellular carcinoma with portal vein tumor thrombosis: the emerging role for radioembolization using yttrium-90. Oncology. 2013;84(5):311-318.

38. Lau WY, Ho S, Leung TW, et al. Selective internal radiation therapy for nonresectable hepatocellular carcinoma with intraarterial infusion of 90yttrium microspheres. Int J Radiat Oncol Biol Phys. 1998;40(3): $583-592$

39. Sangro B, Bilbao JI, Boan J, et al. Radioembolization using 90Y-resin microspheres for patients with advanced hepatocellular carcinoma. Int J Radiat Oncol Biol Phys. 2006;66(3):792-800.

OncoTargets and Therapy

\section{Publish your work in this journal}

OncoTargets and Therapy is an international, peer-reviewed, open access journal focusing on the pathological basis of all cancers, potential targets for therapy and treatment protocols employed to improve the management of cancer patients. The journal also focuses on the impact of management programs and new therapeutic agents and protocols on
40. Sangro B, Iñarrairaegui M, Bilbao JI. Radioembolization for hepatocellular carcinoma. J Hepatol. 2012;56(2):464-473.

41. Riaz A, Kulik L, Lewandowski RJ, et al. Radiologic-pathologic correlation of hepatocellular carcinoma treated with internal radiation using yttrium-90 microspheres. Hepatology. 2009;49(4):1185-1193.

42. Vilgrain V, Bouattour M, Sibert A, et al. SARAH: a randomized controlled trial comparing efficacy and safety of selective internal radiation therapy (with yttrium-90 microspheres) and sorafenib in patients with locally advanced hepatocellular carcinoma. The International Liver Congress $^{\mathrm{TM}} 2017$ 52nd Annual Meeting of the European Association for the Study of the Liver. J Hepatol. 2017;66(Suppl 1):Abs GS-012.

43. Chow PKH, Gandhi M. Phase III multi-center open-label randomized controlled trial of selective internal radiation therapy (SIRT) versus sorafenib in locally advanced hepatocellular carcinoma: The SIRveNIB study. 2017 ASCO Annual Meeting. J Clin Oncol. 2017;35(Suppl): Abs 4002.

44. Bruix J, Sherman M; American Association for the Study of Liver Diseases. Management of hepatocellular carcinoma: an update. Hepatology. 2011;53(3):1020-1022.

45. Woodall CE, Scoggins CR, Ellis SF, et al. Is selective internal radioembolization safe and effective for patients with inoperable hepatocellular carcinoma and venous thrombosis? J Am Coll Surg. 2009;208(3): $375-382$.

46. Goin JE, Salem R, Carr BI, et al. Treatment of unresectable hepatocellular carcinoma with intrahepatic yttrium 90 microspheres: factors associated with liver toxicities. J Vasc Interv Radiol. 2005;16(2 Pt 1): 205-213.

47. Salem R, Lewandowski RJ, Atassi B, et al. Treatment of unresectable hepatocellular carcinoma with use of $90 \mathrm{Y}$ microspheres (TheraSphere): safety, tumor response, and survival. J Vasc Interv Radiol. 2005;16(12): 1627-1639.

48. Chen SW, Lin LC, Kuo YC, Liang JA, Kuo CC, Chiou JF. Phase 2 study of combined sorafenib and radiation therapy in patients with advanced hepatocellular carcinoma. Int $J$ Radiat Oncol Biol Phys. 2014;88(5):1041-1047.

49. Riaz A, Lewandowski RJ, Kulik LM, et al. Complications following radioembolization with yttrium-90 microspheres: a comprehensive literature review. J Vasc Interv Radiol. 2009;20(9):1121-1130.

50. Lam MG, Banerjee S, Louie JD, et al. Root cause analysis of gastroduodenal ulceration after yttrium-90 radioembolization. Cardiovasc Intervent Radiol. 2013;36(6):1536-1547.

51. Pieper CC, Willinek WA, Thomas D, et al. Incidence and risk factors of early arterial blood flow stasis during first radioembolization of primary and secondary liver malignancy using resin microspheres: an initial single-center analysis. Eur Radiol. 2016;26(8):2779-2789.

52. Edeline J, Gilabert M, Garin E, Boucher E, Raoul JL. Yttrium-90 microsphere radioembolization for hepatocellular carcinoma. Liver Cancer. 2015;4(1):16-25.

53. Furuse J, Ishii H, Nagase M, Kawashima M, Ogino T, Yoshino M. Adverse hepatic events caused by radiotherapy for advanced hepatocellular carcinoma. J Gastroenterol Hepatol. 2005;20(10):1512-1518.

54. Seidensticker R, Seidensticker M, Damm R, et al. Hepatic toxicity after radioembolization of the liver using (90)Y-microspheres: sequential lobar versus hole liver approach. Cardiovasc Intervent Radiol. 2012; 35(5):1109-1118.

\section{Dovepress}

patient perspectives such as quality of life, adherence and satisfaction. The manuscript management system is completely online and includes a very quick and fair peer-review system, which is all easy to use. Visit http://www.dovepress.com/testimonials.php to read real quotes from published authors. 